\title{
Consumo alimentar e sua predição pelos sistemas NRC, CNCPS e BR- Corte, para tourinhos zebuínos confinados ${ }^{1}$
}

\author{
Feed intake and its prediction using the NRC, CNCPS and BR-Corte systems for \\ confined young Zebu bulls
}

\author{
Julimar do Sacramento Ribeiro ${ }^{2 *}$, Marcio Machado Ladeira ${ }^{3}$, Otavio Rodrigues Machado Neto $^{4}$ e Fabrício \\ Rodrigues Campos $^{4}$
}

\begin{abstract}
RESUMO - Objetivou-se avaliar a predição de consumo de matéria seca (CMS) pelos sistemas CNCPS 5.0, NRC e BRCORTE, bem como o consumo de nutrientes por tourinhos zebuínos confinados. Foram utilizados 44 animais em delineamento experimental inteiramente casualizado, sendo: 19 Nelore PO, 7 Nelore LA, 10 Tabapuã PO e 8 Guzerá PO, com peso vivo inicial médio de $394 \pm 39 ; 348 \pm 33 ; 346 \pm 28$ e $340 \pm 30 \mathrm{~kg}$, respectivamente, e idade inicial média de 13 meses. O período experimental foi de 84 dias. O consumo alimentar individual foi obtido com o uso dos indicadores LIPE®, óxido crômico e fibra em detergente ácido indigestível (FDAi). Para comparação do CMS predito pelos sistemas com o observado utilizouse análise de regressão linear. Os animais Nelore LA apresentaram os menores valores observados para CMS $\left(\mathrm{kg} \mathrm{d}^{-1}\right)$. Ao avaliar o CMS em porcentagem do peso vivo (\%PV) e em gramas por quilo de peso vivo metabólico ( $\left.\mathrm{g} \mathrm{kg}^{-1} \mathrm{PM}\right)$, foi detectada diferença entre os grupos genéticos, sendo que os maiores valores ocorreram para os animais Tabapuã PO e Guzerá PO. Os sistemas NRC, CNCPS 5.0 e o BR-CORTE apresentaram subpredição do CMS. Conclui-se que os sistemas estudados não se mostraram adequados para predizer o consumo de matéria seca em tourinhos zebuínos confinados.
\end{abstract}

Palavras-chave: Alimentação dos animais. Guzerá (Zebu). Nelore (Zebu). Tabapuã (Zebu).

\begin{abstract}
The objective of this study was to evaluate the prediction of dry matter intake (DMI) using the CNCPS 5.0, NRC and BR-CUT systems, and of the nutrient intake of confined young Zebu bulls. A completely randomized experimental design of 44 animals as follows was used: 19 Nelore PO, 7 Nelore LA, 10 Tabapua PO e 8 Guzerat PO, with a mean initial live body weight of $394 \pm 39,348 \pm 33,346 \pm 28$ and $340 \pm 30 \mathrm{~kg}$ respectively, and a mean initial age of 13 months. The length of the experiment was 84 days. Individual food consumption was calculated with the use of LIPE ®, chromium oxide and indigestible acid detergent fiber (iADF) indicators. In order to compare the DMI as predicted by the systems to that observed, linear regression analysis was used. The Nellore LA group showed the lowest observed values for DMI $\left(\mathrm{kg} \mathrm{d}^{-1}\right)$. When assessing DMI as a percentage of live body weight (\% BW) and in grams per kilogram of metabolic live weight $\left(\mathrm{g} \mathrm{kg}^{-1} \mathrm{MW}\right)$, a difference between genetic groups was found, with the highest values being observed for the Tabapua PO and Guzerat PO groups. The NRC, CNCPS 5.0 and BR-CUT systems under predicted the DMI. It can be concluded that the systems studied were not adequate for the prediction of the dry matter intake of confined young Zebu bulls.
\end{abstract}

Key words: Animal feed. Guzerat (Zebu). Nellore (Zebu). Tabapua (Zebu).

\footnotetext{
*Autor para correspondência

${ }^{1}$ Recebido para publicação em 01/08/2011; aprovado em 03/05/2012

Parte da Dissertação do primeiro autor apresentada ao Programa de Pós-Graduação em Zootecnia da UFLA

${ }^{2}$ Departamento de Zootecnia/UFAL, Campus Arapiraca, s/n, Arapiraca-AL, Brasil, 57.309-005 julimarzoo@ hotmail.com

${ }^{3}$ Departamento de Zootecnia/UFLA, Campus Universitário, s/n, Lavras-MG, Brasil, 37.200-000, mladeira@dzo.ufla.br

${ }^{4}$ Programa de Pós Graduação em Zootecnia/PPGZ/UFLA, Campus Universitário, s/n, Lavras-MG, Brasil, 37.200-000, otavionetto@terra.com.br, fabriciotheba@yahoo.com.br
} 


\section{INTRODUÇÃO}

Informações sobre o consumo de matéria seca (CMS) pelos animais são de extrema importância em todo o processo produtivo, já que o desempenho animal é função direta do consumo de matéria seca digestível. Isso ocorre pela influência direta no ingresso de nutrientes, principalmente, energia e proteína, necessários ao atendimento das exigências de mantença e produção.

Vários estudos têm apresentado relatos sobre diferenças do comportamento ingestivo entre espécies e raças de animais domésticos. De acordo com Eggert e Nielsen (2006), após serem descontadas as possíveis diferenças decorrentes da taxa e da composição do ganho de peso, a variação genética no CMS parece ser devido, basicamente, às diferenças nos gastos energéticos relacionados à mantença. Por isso, pesquisas foram desenvolvidas no Brasil comparando as diferenças no consumo de alimentos entre animais Bos taurus, Bos indicus e seus mestiços (ALVES et al., 2004; EUCLIDES FILHO; FIGUEREDO; EUCLIDES, 2003). Padrões distintos de consumo entre estes animais, mantidos em confinamento, foram bem evidenciados por Almeida et al. (2003), que encontraram consumos mais baixos em zebuínos, comparados aos taurinos e mestiços.

Putrino et al. (2007) verificaram que tourinhos Nelore e Brangus alimentados com diferentes proporções de concentrado (20;40;60 e 80\% de concentrado ou 61,$4 ; 64,1$; 67,05 e 73,0\% de NDT), têm curvas de consumo distintas. No trabalho citado, tourinhos Brangus apresentaram consumo máximo para a dieta contendo $67,05 \%$ de NDT, enquanto que tourinhos Nelore apresentaram consumo máximo para dieta com $64,10 \%$ de NDT.

De acordo com Almeida et al. (2003), a melhor estratégia para a melhoria da predição do consumo de matéria seca é o desenvolvimento de equações específicas para zebuínos. A maioria dos trabalhos realizados no Brasil que avaliaram o CMS de bovinos foram realizados em baias individuais, no entanto devido à interação entre o comportamento animal e o CMS, torna-se necessário estudaraingestãode matéria secaem condições semelhantes àquelas de campo. Para isso, é necessária a utilização de técnicas que possibilitem determinar esses valores de forma precisa, pois o grau de exatidão de suas estimativas possui importância capital (NATIONAL RESEARCH COUNCIL, 2000). Nas condições brasileiras, a técnica dos indicadores consiste na alternativa mais viável para determinação do CMS e vem sendo amplamente empregada para animais em pastejo ou alimentados em grupo, e se baseia na obtenção da massa consumida por meio da relação entre a excreção fecal e a digestibilidade da dieta (MACHADO et al., 2011).
Os fatores que interferem no CMS pelos animais são tratados de forma diferente pelos diversos sistemas de exigências nutricionais, o que gera predições de consumo diferenciadas. Dentre os principais sistemas estão o Nutrient Requeriments of Beef Cattle (NATIONAL RESEARCH COUNCIL, 2000) e o Cornell Net Carbohydrate and Protein System - CNCPS 5.0 (FOX et al., 2003), ambos desenvolvidos sob condições ambientais, de manejo, de alimentação e genéticas diferentes das encontradas no Brasil. Devido a isto, a Universidade Federal de Viçosa desenvolveu o sistema BR-CORTE - Exigências nutricionais de zebuínos (VALADARES FILHO; PAULINO; MAGALHÃES, 2006a), o qual utiliza bancos de dados e materiais genéticos representativos das condições brasileiras, com a finalidade de aumentar a precisão dos valores preditos de consumo.

Objetivou-se avaliar o consumo de matéria seca predito pelos sistemas National Research Council (2000), CNCPS 5.0 e BR-CORTE, bem como o consumo de nutrientes por tourinhos de diferentes grupos genéticos zebuínos.

\section{MATERIAL E MÉTODOS}

Foram utilizados 44 tourinhos de quatro grupos genéticos, em delineamento estatístico inteiramente casualizado, sendo: 19 Nelore PO, 7 Nelore LA, 10 Tabapuã PO e 8 Guzerá PO, com peso vivo inicial médio e desvio padrão de $394 \pm 39,348 \pm 33,346 \pm 28$ e $340 \pm 30 \mathrm{~kg}$, respectivamente, e idade inicial média de 13 meses. Os animais foram confinados em baias coletivas com área de $30 \mathrm{~m}^{2}$ por animal. A instalação de confinamento era de piso de terra compactado e com área próxima ao comedouro em concreto. Os bebedouros coletivos localizavam-se na divisória entre duas baias. O comedouro utilizado foi do tipo vinilona, disposto transversalmente na parte superior da baia, com $70 \mathrm{~cm}$ para cada animal.

Buscando atenuar os efeitos do ganho compensatório sobre o CMS, os animais receberam a mesma dieta e manejo, por um período de 28 dias, sendo esse considerado o período de adaptação. De acordo com a metodologia utilizada, visando a obtenção de valores representativos do CMS para todo o período experimental, a coleta de dados foi feita em dois períodos de 28 dias cada.

A dieta foi balanceada para atender às exigências de ganho de $1,3 \mathrm{~kg} \mathrm{dia}^{-1}$ de acordo com o National Research Council (2000), com uma relação volumoso: concentrado de 30:70 (Tabela 1), relação utilizada em boa parte dos confinamentos brasileiros, de acordo com Millen et al. (2009).

A dieta, em forma de ração total, foi fornecida ad libitum aos animais às $8 \mathrm{~h}$ e $15 \mathrm{~h}$, sendo as sobras coletadas 
Tabela 1 - Composição percentual de ingredientes e bromatológica da dieta fornecida a novilhos zebuínos confinados

\begin{tabular}{|c|c|}
\hline Ingredientes & Composição percentual (\%MS) \\
\hline Silagem de milho & 30,0 \\
\hline Milho integral moído & 25,2 \\
\hline Polpa cítrica & 25,2 \\
\hline Farelo de soja & 16,1 \\
\hline Fosfato bicálcico & 0,5 \\
\hline Bicarbonato de sódio & 0,9 \\
\hline Núcleo mineral $^{1}$ & 2,1 \\
\hline \multicolumn{2}{|c|}{ Composição bromatológica } \\
\hline Matéria seca $(\%)$ & 71,7 \\
\hline Proteína bruta (\% na MS) & 13,0 \\
\hline Fibra em detergente neutro (\% na MS) & 33,2 \\
\hline $\mathrm{CNF}(\%$ na MS) & 48,0 \\
\hline $\mathrm{EE}(\%$ na MS) & 3,3 \\
\hline NDT2 (\% na MS) & 72,0 \\
\hline
\end{tabular}

${ }^{1}$ Níveis de garantia por quilo do produto: Ca: 235g; P: 45g; S: 23g; Na: 80,18g; Zn: 2,38 mg; Cu: 625 mg; Fe: 1,18 mg; Mn: 312 mg; Co: 32 mg; I: 41 ,6 mg; Se: 11,25mg; Vit.A: 70.000 UI; Vit. D3: 5.000 UI; Vit. E: 15 UI; Niacina: 3,33 mg ${ }^{2}$ Calculado segundo o National Research Council (2000)

na manhã seguinte. A quantidade de ração fornecida foi ajustada para permitir sobras de, no mínimo, $5 \%$. A cada 14 dias, foram coletadas amostras da dieta fornecida, do concentrado, da silagem, dos ingredientes do concentrado e das sobras, das quais foram realizadas as análises bromatológicas segundo Silva e Queiroz (2002).

Para a estimação da excreção de matéria seca fecal (EF) foi utilizado o indicador LIPE®, em uma posologia de uma cápsula de $0,5 \mathrm{~g} \mathrm{~d}^{-1}$ por animal (SALIBA et al., 2003), durante os dias 24; 25 e 26 de cada período experimental.

As coletas de fezes foram feitas segundo Ribeiro et al. (2009) conforme o seguinte procedimento: às $10 \mathrm{~h} \mathrm{e}$ 18 hora do dia 25 , às 08 hora e 16 hora do dia 26 e às 06 hora e 14 hora do dia 27. Estas amostras foram pré-secas em estufa com ventilação forçada a $65^{\circ} \mathrm{C}$ por 72 horas e moídas em moinho com peneira de malha de $1 \mathrm{~mm}$, sendo então elaborada uma amostra composta por animal, em cada período, com base no peso seco de cada coleta.

Para avaliar a estabilização da excreção do indicador nas fezes, também foram feitas amostras compostas, correspondendo aos períodos de 24; 48 e 72 horas após o fornecimento do LIPE ${ }^{\circledR}$.

As amostras de fezes foram enviadas à Escola de Veterinária da Universidade Federal de Minas Gerais, para que fossem realizadas as análises do LIPE® de acordo com metodologia descrita por Saliba et al. (2003).

O CMS do concentrado foi estimado utilizando-se o óxido crômico misturado à dieta, na proporção de $10 \mathrm{~g} \mathrm{~d}^{-1}$ por animal e fornecido aos animais entre o 17으 e 27으 dia de cada período, sendo sete dias de adaptação e três dias de coleta de fezes (VALADARES FILHO, 2006b).

Para a determinação do consumo do volumoso utilizou-se a MSi, obtida após 240 horas de incubação ruminal, de acordo com a técnica descrita por Casali et al. (2008). Os cálculos dos consumos foram realizados segundo Valadares Filho et al. (2006b).

Os dados referentes às predições de consumo de matéria seca nos sistemas National Research Council (2000) e CNCPS 5.0 foram obtidos por meio do software específico de cada sistema. Adotaram-se, como peso à maturidade $540 \mathrm{~kg}$ (NATIONAL RESEARCH COUNCIL, 2000; SILVA; LEME; PEREIRA., 2003), condição corporal 6 em uma escala de 1 a 9, temperatura de $24^{\circ} \mathrm{C}$ e umidade relativa de $50 \%$. A dieta avaliada foi aquela obtida por meio do consumo de forragem e concentrado apresentado por cada animal.

A avaliação da exatidão das estimativas do consumo de matéria seca pelos sistemas de exigências nutricionais foi conduzida por meio do ajustamento de modelo de regressão linear simples de valores observados (variável dependente) sobre valores preditos (variável independente) e os testes estatísticos foram conduzidos sob as seguintes hipóteses:

$\mathrm{H}_{0}: \beta_{0}=0$ e $\beta_{1}=1 \mathrm{X} \mathrm{H}_{\mathrm{a}}: \mathrm{naoH}_{0}$

Os valores preditos foram plotados no eixo $\mathrm{X}$ enquanto que os observados no eixo $\mathrm{Y}$, conforme preconizado por Tedeschi (2006). 
No caso de não-rejeição da hipótese de nulidade, concluiu- se pela similaridade entre valores preditos e observados. Aavaliação comparativa da eficiência de predição entre os sistemas foi realizada pela avaliação e decomposição do quadrado médio dos erros de predição (QMEP), segundo protocolos descritos por Kobayashi e Salam (2000).

Para avaliar a estabilização na excreção do indicador, os valores de estimação da produção fecal encontrados, para 24; 48 e 72 horas após o fornecimento do LIPE® foram comparados entre si. As análises estatísticas foram feitas por meio do procedimento GLM do software estatístico SAS (1999) e as médias comparadas pelo teste Scott e Knott.

\section{RESULTADOS E DISCUSSÃO}

Não houve diferença $(\mathrm{P}>0,05)$ na estimativa de produção fecal entre os intervalos de coleta de fezes, 24; 48 e 72 horas após o fornecimento do LIPE $^{\circledR}$ (Figura 1), o que sugere a estabilização da excreção do indicador LIPE ${ }^{\circledR}$ a partir de 24 horas.

Figura 1 - Comparação de três períodos de coletas de fezes para estimar a produção fecal por meio do indicador LIPE ${ }^{\circledR}$. Médias seguidas pela mesma letra não diferem estatisticamente pelo teste Scott e Knott $(\mathrm{P}>0,05)$

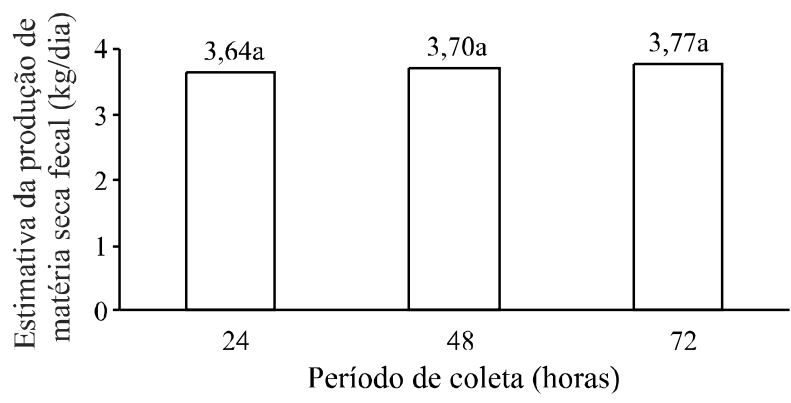

Ferreira et al. (2009) estimaram a produção fecal usando os indicadores LIPE® e óxido crômico e compararam seus resultados com a coleta total de fezes realizada em dois intervalos, três e cinco dias, não encontrando diferenças entre os indicadores e a coleta total, além de também não terem verificado diferenças entre os intervalos de coletas.

Neste experimento, o período de coleta de fezes foi de três dias, entretanto os resultados encontrados podem ser indicativos da possibilidade de fornecimento do LIPE $^{\circledR}$ por período mais curto sem prejudicar a estimativa de produção fecal. Este resultado pode ser importante para a estimativa do CMS em condições de campo, além de mostrar a estabilização do indicador já nas primeiras 24 horas, pois a produção fecal se manteve semelhante durante os dias das coletas. Este fato indica que a estimativa do consumo de matéria seca não foi influenciada pelo manejo dos animais, já que estes eram levados duas vezes por dia ao curral de manejo para coleta de fezes.

Os animais Nelore LA apresentaram menor CMS, expresso em quilogramas por dia, tendo os animais dos demais grupos (Nelore PO, Tabapuã PO e Guzerá PO) apresentado valores semelhantes entre si (Tabela 2).

Quando se compara os animais que tiveram CMS em kg dia ${ }^{-1}$ semelhantes (Nelore PO, Tabapuã PO e Guzerá PO), observa-se que o grupo Nelore PO apresentou CMS ( $\mathrm{g} \mathrm{kg}^{-1} \mathrm{PM}$ ), menor que os demais, o que pode ter ocorrido devido ao maior peso vivo apresentado por esses animais. Esses valores podem indicar que animais Nelore PO apresentam menor gasto com energia de mantença, quando comparados aos Tabapuã PO e Guzerá PO, para uma mesma idade.

Os animais Nelore PO, Nelore LA, Tabapuã PO e Guzerá PO apresentaram peso vivo final de 445; 402; 397 e $393 \mathrm{~kg}$, respectivamente, sendo o peso vivo

Tabela 2 - Médias e respectivos erros padrões do consumo de matéria seca (CMS) por grupos genéticos zebuínos confinados

\begin{tabular}{|c|c|c|c|c|c|c|c|c|c|}
\hline \multirow{2}{*}{ Item } & \multicolumn{9}{|c|}{ Raças } \\
\hline & N. $\mathrm{PO}^{1}$ & E P & N. LA ${ }^{2}$ & E P & Tabapuã & E P & Guzerá & E P & $\mathrm{P}$ \\
\hline CMS $\left(\mathrm{kg} \mathrm{dia}^{-1}\right)$ & $8,74 \mathrm{a}$ & 0,29 & $7,51 \mathrm{~b}$ & 0,48 & $9,61 \mathrm{a}$ & 0,40 & $8,87 \mathrm{a}$ & 0,45 & 0,01 \\
\hline CMS (\% PV) & $2,09 \mathrm{~b}$ & 0,08 & $2,01 \mathrm{~b}$ & 0,13 & $2,60 \mathrm{a}$ & 0,11 & $2,44 \mathrm{a}$ & 0,12 & $<0,01$ \\
\hline $\mathrm{CMS}\left(\mathrm{g} \mathrm{kg}^{-1} \mathrm{PM}^{3}\right)$ & $92,37 \mathrm{~b}$ & 3,33 & $85,40 \mathrm{~b}$ & 5,49 & $110,43 \mathrm{a}$ & 4,59 & $103,47 \mathrm{a}$ & 5,13 & $<0,01$ \\
\hline $\mathrm{CMSc}^{4}\left(\mathrm{~kg} \mathrm{dia}^{-1}\right)$ & $5,93 \mathrm{~b}$ & 0,31 & $5,03 \mathrm{~b}$ & 0,51 & $7,32 \mathrm{a}$ & 0,43 & $5,96 \mathrm{~b}$ & 0,48 & 0,01 \\
\hline $\operatorname{CMSf}^{5}\left(\mathrm{~kg} \mathrm{dia}^{-1}\right)$ & 2,81 & 0,14 & 2,47 & 0,23 & 2,29 & 0,19 & 2,91 & 0,21 & 0,15 \\
\hline $\mathrm{CMSc}^{4}(\%$ da MS $)$ & $67,51 \mathrm{~b}$ & 2,21 & $67,41 b$ & 3,64 & $75,50 \mathrm{a}$ & 3,05 & $67,18 \mathrm{~b}$ & 3,41 & 0,03 \\
\hline $\operatorname{CMSf}^{5}(\%$ da MS $)$ & $32,49 \mathrm{a}$ & 1,77 & $32,59 \mathrm{a}$ & 2,92 & $24,50 \mathrm{~b}$ & 2,44 & $32,82 \mathrm{a}$ & 2,73 & 0,05 \\
\hline
\end{tabular}

${ }^{1}$ N. PO: Nelore puro de origem (PO); ${ }^{2} \mathrm{~N}$. LA: Nelore livro aberto (LA). ${ }^{3} \mathrm{PM}$ : tamanho metabólico; ${ }^{4} \mathrm{CMSc}$ : CMS de concentrado; ${ }^{5} \mathrm{CMSf}$ : CMS de forragem. EP: erro padrão da média. Médias nas linhas, seguidos de mesma letra, não apresentam diferença significativa, pelo teste Scott e Knott $(\mathrm{P}<0,05)$ 
médio durante o período experimental de 419; 375; 372 e $367 \mathrm{~kg}$, respectivamente.

O CMS expresso em porcentagem do peso vivo $(\%$ PV) foi maior para os animais Tabapuã PO e Guzerá PO, em relação aos animais Nelore (PO e LA). O mesmo ocorreu quando o CMS foi corrigido para peso metabólico (PM).

Apesar dos animais Nelore LA terem apresentado CMS diário menor que os animais Nelore PO $(7,51$ e 8,74 kg, respectivamente), os consumos expressos em \% PV e g kg-1 PM foram semelhantes. Isso ocorreu devido à correção da diferença no peso vivo médio de cada grupo, 375 e 419 kg nos Nelore LA e PO, respectivamente.

Os valores de CMS observados ficaram próximos aos relatados por Almeida et al. (2003) $\left(7,6 \mathrm{~kg} \mathrm{dia}^{-1}\right)$ em experimento onde os animais foram submetidos a dieta com relação volumoso:concentrado de 45:55 e por Machado Neto (2010) (10,6 kg dia-1) onde a relação volumoso:concentrado foi de 50:50, sendo ambos os trabalhos realizados com animais da raça Nelore.

Levando em consideração os dados obtidos no presente experimento, o valor médio do CMS, em \% PV $(2,28)$, foi próximo ao descrito por Valadares Filho (2006a) e Miotto et al. (2009), que observaram consumo em torno de 2,20\% PV para animais com características similares aos desse experimento.

O consumo de proteína bruta (CPB) seguiu o comportamento de ingestão de matéria seca, ou seja, os animais da raça Tabapuã apresentaram consumo maior de PB em kg dia-1 $(1,42)$, \%PV $(0,38)$ e $\mathrm{g} \mathrm{kg}^{-1}$ PM $(16,86)$, quando comparados aos demais grupos genéticos (Tabela 3 ).
O CPB médio situou-se próximo, contudo acima dos valores estimados pelo Sistema de Exigências Nutricionais de Zebuínos BR-CORTE (VALADARES FILHO et al., 2006a) e por Silva et al. (2003) (0,95 e $0,94 \mathrm{~kg} \mathrm{dia}^{-1}$, respectivamente). Segundo Valadares Filho et al. (2006a), o CPB apresentado por todos os grupos seria suficiente para garantir um ganho de peso diário em torno de $1,25 \mathrm{~kg} \mathrm{dia}^{-1}$.

De acordo com a equação proposta por Detmann et al. (2003), para animais em confinamento, a qual considera a proporção de volumoso na dieta $(30 \%$ da matéria seca, no presente experimento) para predizer o consumo de fibra, os animais deveriam apresentar um consumo de fibra em detergente neutro (CFDN) médio igual a $2,55 \mathrm{~kg}$, valor próximo ao observado $(2,46 \mathrm{~kg})$. Ademais, os valores observados ficaram abaixo do proposto por Mertens (1992), o qual estabeleceu que o consumo de FDN em níveis acima de 1,2\% do peso corporal do animal seria um dos principais mecanismos físicos reguladores do consumo de MS, o que representaria nas condições do experimento um CFDN de 4,80 kg, portanto, a dieta não proporcionou limitação física ao CMS. Pelo contrário, como a dieta apresentava 70\% de concentrado, o mecanismo de regulação energética de consumo, provavelmente, deveria estar atuando, o que é de se esperar no sistema de alimentação em confinamento. Nessa situação, de acordo com Silva (2006), um aporte maior de metabólitos estaria chegando à corrente sanguínea, principalmente o propionato, exercendo um controle químico sobre o sistema nervoso central (SNC), inibindo o consumo.

O consumo de extrato etéreo apresentado por estes animais foi, em média, igual a $0,28 \mathrm{~kg} \mathrm{dia}{ }^{-1}$,

Tabela 3 - Médias e respectivos erros padrões do consumo de proteína bruta (CPB), de fibra em detergente neutro (CFDN) e de extrato etéreo (CEE) por grupos genéticos zebuínos confinados

\begin{tabular}{lccccccccc}
\hline \multirow{2}{*}{ Item } & \multicolumn{10}{c}{ Raças } \\
\cline { 2 - 10 } & N. PO & E P & N. LA & E P & Tabapuã & E P & Guzerá & E P & P \\
\hline CPB $\left(\mathrm{kg} \mathrm{dia}^{-1}\right)$ & $1,21 \mathrm{~b}$ & 0,05 & $1,03 \mathrm{~b}$ & 0,08 & $1,42 \mathrm{a}$ & 0,07 & $1,16 \mathrm{~b}$ & 0,08 & 0,01 \\
$\mathrm{CPB}(\% \mathrm{PV})$ & $0,29 \mathrm{~b}$ & 0,01 & $0,28 \mathrm{~b}$ & 0,02 & $0,38 \mathrm{a}$ & 0,02 & $0,32 \mathrm{~b}$ & 0,02 & $<0,01$ \\
$\mathrm{CPB}\left(\mathrm{g} \mathrm{kg}^{-1} \mathrm{PM}^{3}\right)$ & $13,06 \mathrm{~b}$ & 0,62 & $12,15 \mathrm{~b}$ & 1,03 & $16,86 \mathrm{a}$ & 0,86 & $13,97 \mathrm{~b}$ & 0,96 & $<0,01$ \\
$\mathrm{CFDN}\left(\mathrm{kg} \mathrm{dia}^{-1}\right)$ & 2,62 & 0,08 & 2,27 & 0,13 & 2,58 & 0,08 & 2,38 & 0,09 & 0,13 \\
$\mathrm{CFDN}\left(\% \mathrm{PV}^{2}\right)$ & 0,63 & 0,02 & 0,60 & 0,04 & 0,70 & 0,03 & 0,65 & 0,04 & 0,27 \\
$\mathrm{CFDN}\left(\mathrm{g} \mathrm{kg}^{-1} \mathrm{PM}^{3}\right)$ & 28,30 & 0,8 & 26,67 & 0,98 & 30,54 & 0,89 & 28,67 & 0,91 & 0,35 \\
$\mathrm{CEE}\left(\mathrm{kg} \mathrm{dia}^{-1}\right)$ & $0,29 \mathrm{a}$ & 0,01 & $0,25 \mathrm{~b}$ & 0,02 & $0,32 \mathrm{a}$ & 0,01 & $0,27 \mathrm{~b}$ & 0,02 & 0,02 \\
$\mathrm{CEE}\left(\% \mathrm{PV}^{2}\right)$ & $0,069 \mathrm{~b}$ & 0,003 & $0,067 \mathrm{~b}$ & 0,005 & $0,087 \mathrm{a}$ & 0,004 & $0,075 \mathrm{~b}$ & 0,004 & $<0,01$ \\
$\mathrm{CEE}\left(\mathrm{g} \mathrm{kg}^{-1} \mathrm{PM}^{3}\right)$ & $3,14 \mathrm{~b}$ & 0,12 & $2,94 \mathrm{~b}$ & 0,20 & $3,80 \mathrm{a}$ & 0,17 & $3,29 \mathrm{~b}$ & 0,19 & $<0,01$ \\
\hline
\end{tabular}

${ }^{1}$ N. PO: Nelore puro de origem (PO); ${ }^{2}$ N. LA: Nelore livro aberto (LA); ${ }^{3}$ PM: peso metabólico. EP: erro padrão da média. Médias nas linhas, seguidos de mesma letra, não apresentam diferença significativa, pelo teste de Scott Knott $(\mathrm{P}<0,05)$ 
sendo que as diferenças observadas para o consumo deste componente da dieta é resultado das diferenças observadas para o consumo de matéria seca, uma vez que todos os animais experimentais foram submetidos à mesma dieta.

Pode-se observar que houve uma variação (Tabela 4) entre o consumo de matéria seca $\left(\mathrm{kg} \mathrm{d}^{-1}\right)$ observado nos animais experimentais e predito pelos sistemas BR-CORTE, CNCPS 5.0 e National Research Council (2000).

Para todos os sistemas avaliados houve rejeição da hipótese de nulidade (Figura 2; 3 e 4, e Tabela 5), ou seja, o intercepto foi diferente de 0 e a inclinação diferente de 1 , dessa forma, os valores preditos (Tabela 4) pelos sistemas de exigências nutricionais diferiram estatisticamente dos valores observados.

Tabela 4 - Consumo mínimo, médio e máximo de matéria seca, observados e preditos pelos sistemas BR-CORTE, CNCPS 5.0 e National Research Council (2000)

\begin{tabular}{|c|c|c|c|c|c|c|}
\hline \multicolumn{7}{|c|}{ Consumo de matéria seca $\left(\mathrm{kg} \mathrm{dia}^{-1}\right)$} \\
\hline & \multirow[b]{2}{*}{ Observado } & \multirow[b]{2}{*}{ EP } & \multirow[b]{2}{*}{$\mathrm{N}$} & \multicolumn{3}{|c|}{ Predito } \\
\hline & & & & $\begin{array}{c}\text { BR- } \\
\text { CORTE }\end{array}$ & CNCPS & NRC \\
\hline Mínimo & 6,41 & 0,19 & 44 & 6,45 & 6,10 & 7,18 \\
\hline Médio & 8,85 & 0,34 & 44 & 8,58 & 7,85 & 8,29 \\
\hline Máximo & 11,20 & 0,24 & 44 & 10,61 & 8,80 & 9,34 \\
\hline
\end{tabular}

Figura 2 - Consumo de matéria seca observado versus consumo predito pelo sistema BR-CORTE. Linha contínua representa valores da reta de equidade $(\mathrm{Y}=\mathrm{X})$; pontos e linha tracejada representam a relação obtida entre os valores observados e preditos

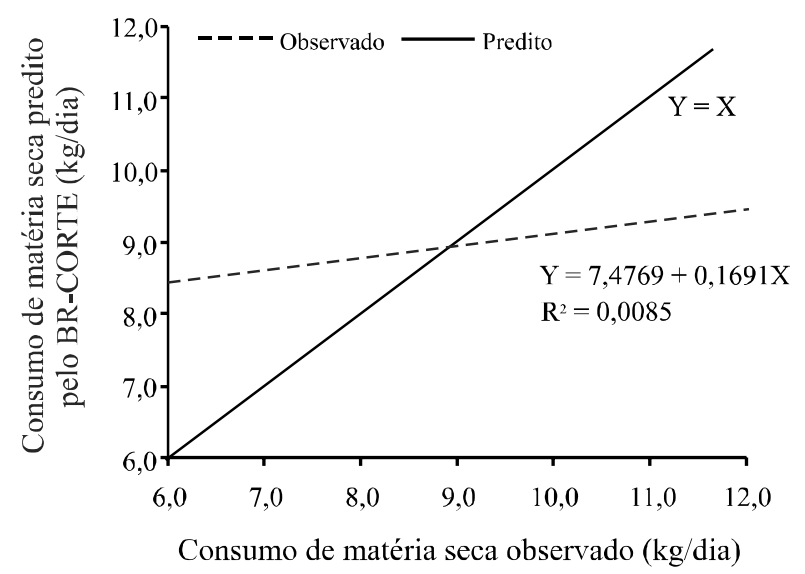

Figura 3 - Consumo de matéria seca observado versus consumo predito pelo sistema CNCPS. Linha contínua representa valores da reta de equidade $(\mathrm{Y}=\mathrm{X})$; pontos e linha tracejada representam a relação obtida entre os valores observados e preditos

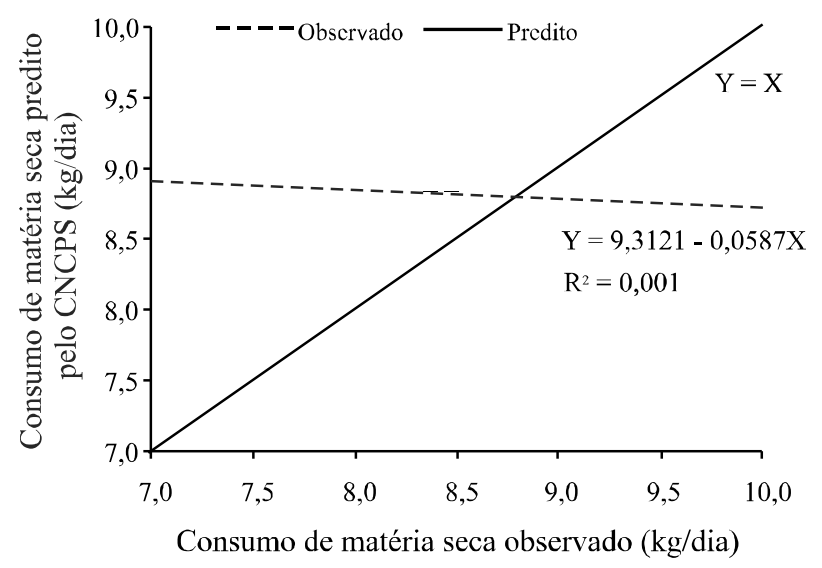

Figura 4 - Consumo de matéria seca observado versus consumo predito pelo sistema NRC. Linha contínua representa valores da reta de equidade $(\mathrm{Y}=\mathrm{X})$; pontos e linha tracejada representam a relação obtida entre os valores observados e preditos

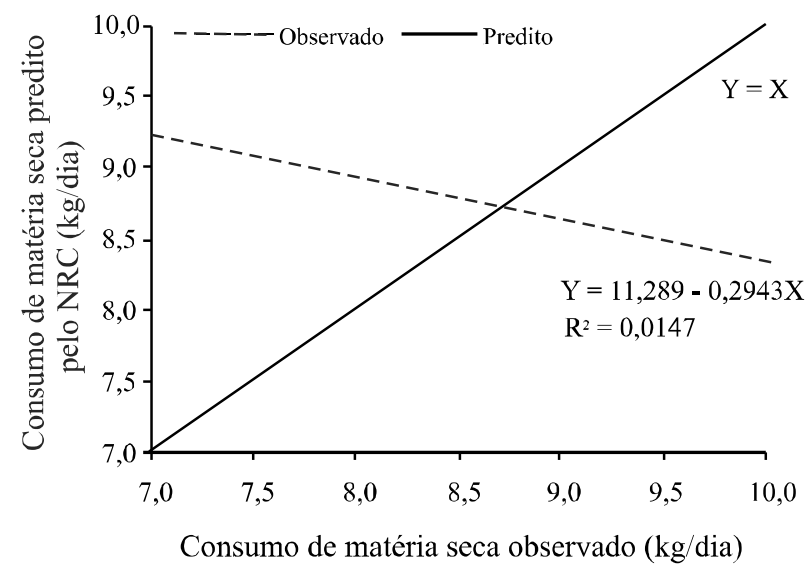

Segundo Kobayashi e Salam (2000), a técnica de análise de regressão apresenta limitações na comparação de valores observados e preditos por modelos matemáticos, pois ao se utilizar esta técnica, assume-se que existe relação linear entre os valores preditos e observados, o que nem sempre é verdadeiro, pois pode haver relação curvilínea entre valores preditos e observados. Por isso, segundo Tedeschi (2006), este tipo de avaliação deve ser baseada na análise do quadrado médio do erro de predição (QMEP). De acordo com os resultados obtidos após análise do QMEP e seus 
Tabela 5 - Análise de regressão dos valores de consumo de matéria seca (CMS) observados e preditos pelos sistemas BRCORTE, CNCPS E NRC

\begin{tabular}{lccccc}
\hline \multicolumn{1}{r}{ Sistema } & $\mathrm{R}_{\mathrm{a}}^{2}$ & $\mathrm{a}$ & $\mathrm{B}$ & $\mathrm{EP}$ & $\mathrm{P}$ \\
\hline BR-CORTE & $-0,1265$ & 7,4744 & 0,1694 & 1,7103 & $<0,01$ \\
CNCPS & $-0,0233$ & 9,3120 & $-0,0587$ & 1,7233 & $<0,01$ \\
NRC & $-0,0093$ & 11,2891 & $-0,2942$ & 1,6998 & $<0,01$ \\
\hline
\end{tabular}

componentes, observa-se que os sistemas NRC e BRCORTE apresentaram valores preditos mais próximos aos observados quando comparados ao CNCPS, já que estes sistemas apresentaram menores valores de desvio médio e sistemático (Tabela 6). Apesar disso, levando em consideração a análise de regressão (Tabela 5) e a análise de QMEP (Tabela 6), nenhum dos sistemas foram eficientes na predição do consumo de matéria seca.

Machado Neto et al. (2010) avaliaram as predições de consumo pelos sistemas NRC, CNCPS e BR-CORTE, verificando que, como no presente trabalho, os sistemas não foram eficientes na predição do consumo de matéria seca. Entretanto, o autor verificou, após análise do QMEP, que o sistema com predições mais aproximadas foi o CNCPS. No entanto, este autor trabalhou com animais do grupo genético Red Norte (3/4 Bos taurus), em sistema de confinamento. Considerando que o banco de dados dos sistemas NRC e CNCPS é composto amplamente por animais taurinos, é de se esperar que estes sistemas possam ser mais eficientes na predição da ingestão de matéria seca por estes animais.

Apesar dos consumos médios observados serem próximo àqueles preditos pelos sistemas, acredita-se que a diferença possa estar relacionada com a elevada dispersão dos dados observados em relação aos dados preditos. Outro fator que pode estar relacionado é a relação volumoso:concentrado da dieta (30:70), pois, Rezende et al. (2011) avaliando as predições dos sistemas NRC e CNCPS concluíram que os sistemas não foram eficientes na predição da ingestão de matéria seca de bovinos submetidos à terminação com $80 \%$ de concentrado na dieta.

Acredita-se, que o efeito negativo da elevação do nível de concentrado na dieta sobre a estimativa de CMS é ocasionada pela alteração no consumo devido à elevação do nível de metabólitos na corrente sanguínea, o que promove maior atuação dos mecanismos de controle químico da ingestão de alimentos, como relatado por Silva (2006). Ademais, segundo Gesualdi Júnior et al. (2005) o ganho compensatório, fenômeno muito comum em rebanhos de países de clima tropical e mais raro em climas temperados, também pode contribuir para a ocorrência de simulações que subpredizem o ganho em peso esperado. Entretanto, a extensão do ganho compensatório, se nulo, parcial ou completo é de difícil predição.

Tabela 6 - Análise do quadrado médio do erro de predição (QMEP) e sua decomposição

\begin{tabular}{lccc}
\hline & \multicolumn{2}{c}{ Sistema } \\
\cline { 2 - 4 } \multicolumn{1}{c}{ QMEP } & NRC & CNCPS & BR-CORTE \\
\cline { 2 - 4 } & 2,40741 & 3,17361 & 2,49742 \\
\hline Variância do QMEP & 6,88742 & 17,20878 & 8,31132 \\
Desvio Padrão do QMEP & 2,62439 & 4,14835 & 2,88294 \\
Coeficiente de Variação do QMEP & $109,01 \%$ & $130,71 \%$ & $115,43 \%$ \\
Raiz Quadrada do QMEP & 1,55518 & 1,78146 & 1,58032 \\
\hline & Decomposição do QMEP & 0,52850 \\
\hline Desvio Médio & 0,32041 & 1,00000 & 0,33815 \\
Desvio Sistemático & 0,13863 & 0,41927 & 1,63078 \\
Erros Aleatórios & 1,62076 & 1,64323 & \\
\hline
\end{tabular}




\section{CONCLUSÕES}

1. Os animais pertencentes aos diferentes grupos genéticos apresentaram diferenças no consumo de matéria seca e de nutrientes, principalmente quando se leva em consideração o tamanho metabólico.

2.Os sistemas BR-CORTE, CNCPS 5.0 e NRC (2000) não se mostraram adequados para predizer o CMS de tourinhos zebuínos em confinamento.

\section{REFERÊNCIAS}

ALMEIDA, R. et al. Influence of genotype on performance and dry matter intake by feedlot steers in Brazil. In: WORLD CONFERENCE ON ANIMAL PRODUCTION, 9.; REUNIÃO DA ASSOCIAÇÃO LATINOAMERICANA DE PRODUÇÃO ANIMAL, 18., 2003. Porto Alegre. Proceedings... Porto Alegre: Associação Latino Americana de Produção Animal, 2003. p. 84.

ALVES, D. D. et al. Desempenho produtivo de bovinos Zebu e cruzados Holandês-Zebu nas fases de recria e terminação, Acta Scientiarum. Animal Science, v. 26, n. 03, p. 385-391, 2004.

CASALI, A. O. et al. Influência do tempo de incubação e do tamanho de partículas sobre os teores de compostos indigestíveis em alimentos e fezes bovinas obtidos por procedimentos in situ. Revista Brasileira de Zootecnia, v. 37, n. 02, p. 335342, 2008.

DETMANN, E. et al. Consumo de fibra em detergente neutro por bovinos em confinamento. Revista Brasileira de Zootecnia, v. 32, n. 06, p. 1763-1777, 2003.

EGGERT, D. L.; NIELSEN, M. K. Comparison of feed energy costs of maintenance, lean deposition, and fat deposition in three lines of mice selected for heat loss. Journal of Animal Science, v. 84, n. 02 , p. $276-282,2006$.

EUCLIDES FILHO, K.; FIGUEREDO, G. R.; EUCLIDES, V. P. B. Desempenho de diferentes grupos genéticos de bovino de corte em confinamento. Revista Brasileira de Zootecnia, v. 32, n. 05, p. 1114-1122, 2003.

FERREIRA, M. A. et al. Avaliação de indicadores em estudos com ruminantes: digestibilidade. Revista Brasileira de Zootecnia, v. 38, n. 08, p. 1568-1573, 2009.

FOX, D. G. et al. The Net Carbohydrate and Protein System for evaluating herd nutrition and nutrient excretion. New York: Cornell University, 2003.

GESUALDI JUNIOR, A. et al. Validação dos sistemas VIÇOSA, CNCPS e NRC para formulação de dietas para bovinos Nelore e Caracu, não-castrados, selecionados em condições brasileiras. Revista Brasileira de Zootecnia, v. 34, n. 03, p. 997-1005, 2005.

KOBAYASHI, K.; SALAM, M. U. Comparing simulated and measured values using mean squared deviation and its components. Agronomy Journal, v. 92, n. 02, p. 345-352, 2000.
MACHADO, A. S. et al. Utilização de óxido crómico e LIPE® como indicadores externos na estimativa de digestibilidade em ruminantes. PUBVET, v. 05, n. 20, 2011. Disponível em: <http://www.pubvet.com.br/artigos_det.asp?artigo=1011>. Acesso em: 13 ago. 2011.

MACHADO NETO, O. R. et al. Feed intake and prediction assessments using the NRC, CNCPS and BR-CORTE systems in Nellore and Red Norte steers finished in feedlot. Revista Brasileira de Zootecnia, v. 39, n. 02, p. 394-401, 2010.

MERTENS, D. R. Análise da fibra e sua utilização na avaliação de alimentos e formulação de rações. In: SIMPÓSIO INTERNACIONAL DE RUMINANTES, 1992, Lavras. Anais... Lavras: SBZ, 1992. p. 188-219.

MILLEN, D. D. et al. A snapshot of management practices and nutritional recommendations used by feedlot nutritionists in Brazil. Journal of Animal Science, v. 87, n. 10, p. $3427-$ 3439, 2009.

MIOTTO, F. R. C. et al. Desempenho de tourinhos Nelore $\mathrm{x}$ Limousin alimentados com dietas contendo gérmen de milho integral. Revista Ciência Agronômica, v. 40, n. 04, p. 624632, 2009.

NATIONAL RESEARCH COUNCIL . Nutrient Requirements of Beef Cattle. 7. Ed. rev. Washington, D. C.: National Academy Press, 2000. 242 p.

PUTRINO, S. M. et al. Digestibilidade aparente de dietas com níveis crescentes de concentrado em novilhos Brangus e Nelore. Arquivo Brasileiro de Medicina Veterinária e Zootecnia, v. 59, n. 02, p. 406-413, 2007.

REZENDE, P. L. P. et al. Validação de modelos matemáticos para predição de consumo voluntário e ganho em peso de bovinos. Archivos de Zootecnia, v. 60, n. 01, p. 2-10, 2011.

RIBEIRO, J. S. et al. Predição de desempenho com uso de sistemas de exigências nutricionais e características de carcaça de tourinhos zebuínos. Pesquisa Agropecuária Brasileira, v. 44, n. 12, p. 1724-1730, 2009.

SALIBA, E. O. S. et al. Lignin from Eucaliptus grandis as indicator for rabbits in digestibility trials. Tropical and Subtropical Agroecosytems, v. 3, n. 1, p. 107-109, 2003.

SILVA, D. J.; QUEIROZ, A. C. Análise de alimentos: métodos químicos e biológicos. Viçosa, MG: UFV, 2002. $235 \mathrm{p}$.

SILVA, J. F. C. da. Mecanismos reguladores de consumo. In: BERCHIELLI, T. T.; PIRES, A. V.; OLIVEIRA, S. G. Nutrição de Ruminantes. Jaboticabal: FUNEP, 2006. p. 57-78.

SILVA, S. L.; LEME, P. R.; PEREIRA, A. S. C. Correlações entre Características de Carcaça Avaliadas por Ultrasom e Pós-abate em Novilhos Nelore, Alimentados com Altas Proporções de Concentrado. Revista Brasileira de Zootecnia, v. 32, n. 05, p. 1236-1242, 2003.

STATISTICAL ANALYSES SYSTEM. User's guide: statistics, versão 8.1. 4. ed., Cary: SAS Institute, 1999. v. 2. 1 CD-ROM. 
TEDESCHI, L. O. Assessment of the adequacy of mathematical models. Agricultural Systems, v. 89, n. 02/03, p. 225-247, 2006.

VALADARES FILHO, S. C.; PAULINO, P. V. R; MAGALHÃES, K.A. Exigências nutricionais de zebuínos e tabelas de composição de alimentos BR-CORTE. Viçosa, MG: UFV/DZO, 2006a. 142 p.
VALADARES FILHO, S. C.; MORAES, E. H. B. K.; DETMANN, E. Perspectivas do uso de indicadores para estimar o consumo individual de bovinos alimentados em grupo. In: REUNIÃO ANUAL DA SOCIEDADE BRASILEIRA DE ZOOTECNIA, 42., 2006, João Pessoa. Anais..., João Pessoa: SBZ, 2006b. 